\title{
Los fitoestrógenos no son eficaces para los calores de la post- menopausia
}

\section{Objetivo}

Evaluar la eficacia y seguridad de suplementos de isoflavona para reducir los calores y mejorar la calidad de vida en las mujeres postmenopáusicas sintomáticas.

\section{Diseño}

Ensayo clínico aleatorizado doble ciego controlado con placebo, con un seguimiento de 12 semanas.

\section{Lugar}

Estudio multicéntrico realizado en 3 centros médicos de Estados Unidos.

\section{Pacientes}

Se incluyeron 252 mujeres postmenopáusicas recientes de 45 a 60 años que experimentaran al menos 35 episodios semanales de sofocos/calores. Fueron excluidas las vegetarianas, las consumidoras de medicaciones que afectan la absorción de isoflavona o de productos de soja más de una vez por semana.

\section{Intervención}

Luego de 2 semanas de período de "run-in"* con placebo, se aleatorizó a las pacientes para recibir promensil (82 $\mathrm{mg}$ de isoflavona), rimostil (57 $\mathrm{mg}$ de isoflavona) o placebo una vez por día.

\section{Medición de resultados principales}

El resultado primario fue la cantidad de episodios diarios de calores. Como resultados secundarios incluyeron los cambios en la calidad de vida mediante la escala de climaterio de Greene, un cuestionario validado para la evaluación de síntomas atribuidos a la menopausia para 4 dominios* (psicosocial, somático, vasomotor y vida sexual), y los efectos adversos.

\section{Resultados principales}

De 252 participantes, 246 (98\%) completaron las 12 semanas de seguimiento. El análisis fue por intención de tratar*. Como se observa en la Tabla, los episodios de calores resultaron similares para las tres ramas al finalizar el estudio. Con respecto a la calidad de vida, se observó una mejoría significativa en los tres grupos a las 12 semanas, pero no hubo diferencias significativas entre los grupos en ningún dominio* de la escala de climaterio de Greene. Tampoco hubo asociación con spotting vaginal, tensión mamaria, trombosis venosa, embolismo pulmonar, infarto de miocardio, accidente cerebrovascular, fracturas o enfermedad de vesícula biliar. No se observaron cambios del peso corporal, presión arterial o frecuencia cardíaca.

Tabla. Efectos del Promensil, Rimostil o placebo en los sofocos.

\begin{tabular}{l|c|c|c}
\multicolumn{1}{|c|}{ Resultados a las 12 semanas } & \multicolumn{1}{c|}{$\begin{array}{c}\text { Promensil } \\
(\mathbf{n}=\mathbf{8 4})\end{array}$} & $\begin{array}{c}\text { Placebo } \\
(\mathbf{n}=527)\end{array}$ & $\begin{array}{c}\text { Placebo } \\
(\mathbf{n}=\mathbf{8 5})\end{array}$ \\
\hline Promedio de sofocos diarios (IC 95\%) & $5,1(4,2-6,0)$ & $5,4(84,4-6,3)$ & $5,0(84,3-5,8)$ \\
\hline $\begin{array}{l}\text { \% de reduccíon del valor basal de } \\
\text { sofocos (IC 95\%) }\end{array}$ & $41 \%(29-51)$ & $34 \%(22-46)$ & $36 \%(26-45)$ \\
\hline
\end{tabular}

\section{Conclusión}

Aunque el estudio mostró alguna evidencia sobre los efectos biológicos del promesil, ningún suplemento demostró una eficacia clínicamente significativa para los calores y otros síntomas de menopausia.

Fuente de financiamiento: Novogen Inc.

\section{Comentario}

Los calores son uno de los motivos de consultas médicas más frecuentes de las mujeres postmenopáusicas. La terapia de reemplazo hormonal (TRH) es muy efectiva, reduciendo un $77 \%$ la frecuencia de calores con respecto al placebo; y también su intensidad. Sin embargo, los recientes resultados de Women's Health Initiative (WHI) y de Heart and Estrogen/progestin Replacement Study (HERS II) han demostrado que el uso de la TRH se asoció con el aumento de riesgo para eventos cardiovasculares, trombóticos y de cáncer de mama. ${ }^{1-3}$ Por lo tanto, la indicación de TRH ha quedado reducida al tratamiento de los síntomas menopáusicos a corto plazo y al tratamiento de la osteoporosis establecida que responde mal a bifosfonatos sólos o asociado a ellos.

Surgieron entonces alternativas para el tratamiento de calores, como los análogos estrogénicos (tibolona), los inhibidores de la recaptación de la serotonina y la isoflavona.

Existen multiples estudios, la mayoría pequeños y con diseños dis- cutibles, que evalúan la eficacia de suplementos de isoflavona para el tratamiento de los síntomas de menopausia, con resultados diferentes. Dos ensayos clínicos recientes que compararon la eficacia de isoflavona derivada de soja con placebo no demostraron diferencias significativas entre ambos grupos, siendo consistente con algunos reportes previos sobre la ineficacia de estos productos. ${ }^{4.5}$

\section{Conclusiones del comentador}

Según la evidencia disponible, las isoflavonas no son eficaces para el tratamiento de los calores postmenopáusicos. Aunque el uso de la TRH a largo plazo cayó en desuso por sus riesgos, sigue siendo el tratamiento más efectivo para controlar los síntomas menopáusicos y se podría usar a corto plazo en mujeres muy sintomáticas.

\section{Dra. Yoo Chu Yon [ Unidad de Medicina Familiar y Preventiva. Hospital Italiano de Buenos Aires. ]}

Chu Yon Y. Los fitoestrógenos no son eficaces para los calores de postmenopausia. Evid actual pract ambul 2005;8:38.Comentado de Tice JA, Ettinger B, Ensrud $\mathrm{K}$ y col. Phytoestrogen supplements for the treatment of hot flashes :the isoflavone clover extract (ICE) study. A randomized controlled trial. JAMA. 2003;290:207-14. PMID: 12851275

\section{Referencias}

1. Writing Group for the Women's Health Initiative Investigators. Risks and benefits of estrogen plus progestin in healthy postmenopausal women: principal results from the Women's Health Initiative randomized controlled trial. JAMA. 2002;288:321-333.

2. Grady D, Herrington D, Bittner V, et al, for the HERS Research Group. Cardiovascular disease outcomes during 6,8 years of hormone therapy: Heart and Estrogen/progestin Replacement Study follow-up (HERS II). JAMA. 2002;288:49-57.

3. Hulley S, Furberg C, Barret-Connor E, et al, for the HERS Research Group. Noncardiovascular disease outcomes during 6,8 years of hormone therapy: Heart and Estrogen/progestin Replacement Study follow-up (HERS II). JAMA. 2002;288:58-66.

4. Penotti M, Fabio E, Modena AB. Effect of soy-derived isoflavones on hot flushes, endometrial thickness, and the pulsatility index of the uterine and cerebral arteries. Fertil Steril 2003;79:1112-1117.

5. Nikander E, Kikkinen A, Metsa-Heikkila M, ET al. A randomized placebo-controlled crossover trial with phytoestrogens in treatment of menopause in breast cancer patients. Obstet Gynecol 2003;101:1213-1220. 